\title{
Study and Analysis of a "Disaster Recovery" Information System using Cloud-computing Technology
}

\author{
Eleni Gialinou \\ Dept. of Industrial Design \\ and Production \\ Engineering, UNI.W.A \\ Athens, Greece
}

\author{
Christos Drosos, PhD \\ Dept. of Industrial Design \\ and Production \\ Engineering, UNI.W.A \\ Athens, Greece
}

\author{
Michail \\ Papoutsidakis, PhD \\ Dept. of Industrial Design \\ and Production \\ Engineering, UNI.W.A \\ Athens, Greece
}

\author{
K. Kalovrektis, PhD \\ Dept. of Informatics \\ University of Thessaly \\ Lamia, Greece
}

\begin{abstract}
The registry of a public service, both in printed and electronic form, is a crucial factor for its operation and therefore for the provided services. Natural and technological disasters are unavoidable and therefore ensuring the recovery of the registry is a very important factor for the operation of the administrative unit. Based on current technological trends, the most appropriate solution is to implement a cloud computing information system, which requires the complete digitization of the file, which consists of paper and electronic forms. The administrative unit, which is under study in the present project, consists of a University secretariat. The ultimate goal of the project is to design an information system that responds to the needs and requirements of the unit, at the lowest possible cost and simultaneously to enable a mechanism for data retrieving at any time, even after a possible disaster. The design of the proposed solution will take into account the use of economical and ecological methods, as well as the relevant IT systems already on the market and specifically those have already implemented in administrative units.
\end{abstract}

\section{Keywords}

Information system, cloud computing, natural disaster, manmade disaster, disaster recovery system, total quality management.

\section{INTRODUCTION}

The number of natural and technological disasters is increasing dramatically on a global level, although it is not fully and systematically has recorded and represented in an international database. Negative consequences confronting at the socio-economic system is improving in the light of the significant progress in forecasting, warning and response in emergency situations. Natural and man-made disasters greatly affect modern society and many people are called to face the effects of a disaster at a professional, administrative and political level.

The more complex is a society on a technological aspect, the greater the risks and the effects of destructive events. However, they can be effectively confronted through objective judgment and action and even more so by implementing systems that have built-in mechanisms for cope with such events. In recent decades, systematic efforts have been done to integrate new technologies into public administration and adapt them to the integration of new management methods and practices (Total Quality Management) and business process reorganizing of administrative units. It is about a continuous process with particular design requirements, so that the implementation of new systems can only have positive effects and this can be verified by appropriate methods.

At the same time, it is well known that in any working environment there is always the danger of natural or manmade disasters (such as sudden power disruption, earthquake, terrorist attacks, etc.). Especially in recent decades, has become perceived that security can be ensured only by establishing a framework of precautionary action. Therefore, efforts are being spent to manage the risk, so that in case of any disaster the company / institute to recover in the least time and with the least losses.

The present paper discusses the study and analysis of the structure and the operation of an administrative unit in the public sector, in order to design an information system for data recovery in case of a natural or technological disaster (disaster recovery information system).

The study of the specific administrative unit (secretariat of University) is selected only to approximate the operation of a public administration unit in real conditions of operation and not the study or analysis of the specific administrative unit. Therefore, it were made efforts so as the proposed information system to be designed with as realistic data as possible. Following a bibliographic research of the theoretical background of the present issue, the existing way of operation of the unit under study as recorded, and the systems are applied to other administration units it was conducted a study for the rudimentary design of a Disaster Recovery Information System.

\subsection{Quality of provided services in Public Administration (Total Quality Management)}

The initial purpose of this paper was to study and analyze the operation of a public sector unit, so as to determine a disaster recovery mechanism for the files in case of a natural or manmade disaster. However, in the stage of the bibliographic review, it was ascertained that for the implementation of a new system in an administrative unit, it is appropriate to take into account the principles of Total Quality Management. In this way, except of the application of the proposed system for the confronting of the basic issue under study, they will be adopted strategies to the direction of overall improvement of unit operation and optimal service provision.

Total Quality Management is a field of the administrative sciences, which deals with the application of methods for the optimal provision of products and services and has recently begun to be applied in the field of public administration in Greece. According to Crosby (1979) [3] "The cost of quality 
is the expense of nonconformance. The cost of quality is that which is avoided if the supply of the product or service is the desired from the beginning". In the present paper, the reference to this issue is triggered by the Prevention Cost, which arises from the key issue (a disaster recovery system) and relates to a prevention action from an event that may occur, but in this case the management of the consequences is vital to the operation of the administration unit.

The prevention cost, in this case, refers to the design and maintain the information system, so that it will actually be able to deliver the desired result. Philip Crosby [16] emphasized in the satisfaction of recipients' (of services) requirements, focusing on prevention rather than correction [16]. Many of the points, Crosby emphasizes in his theories are largely in line with the current provisions of Greek legislation on public administration, where the "Goal setting administration" system is now adopted (Law Number $3230 / 2004$ issue A), where is described the methodology for the efficiency of the public administrative units' operation, so as to respond to modern needs and new management standards.

\subsection{Natural and man-made disasters}

According to E. L. Lekkas "Natural disaster can be a rapid, instantaneous or major collision of the natural environment with the socio-economic system". According to other theories, the phenomenon in detail is defined as "an event in space and time that constitutes a danger for the society or some part of it with serious unintentional consequences as a result of the inadequacy of precautions, which until then were considered adequate"[1].

In general, disasters can be divided into two categories, according to the nature of the reason which caused the phenomenon:

A. Natural disasters: it is about the result from processes that occur in nature, usually without human presence and intervention.

B. Man-made disasters: they arise from human actions directly or indirectly, as a result of technological development, modern way of life and sometimes aggressive behavior of the human factor to the environment [7].

Natural disaster types include phenomena such as flood, fire, earthquake, extreme temperature conditions, biological factor (fungi, rodents, insects) and impact of human factor (technological disasters). Due to the excessive human intervention in the ecosystem, it is obvious that sometimes the boundaries between the two above categories (natural and man-made) are not distinguishable. At the same time, according to the data published on the website "Our world in data" (Source: https://ourworldindata.org/natural-disasters), it is obvious that the number of natural disasters that have occurred in recent decades is dramatically high. This web site presents the data and results of empirical research on global change and in particular global conditions of living.

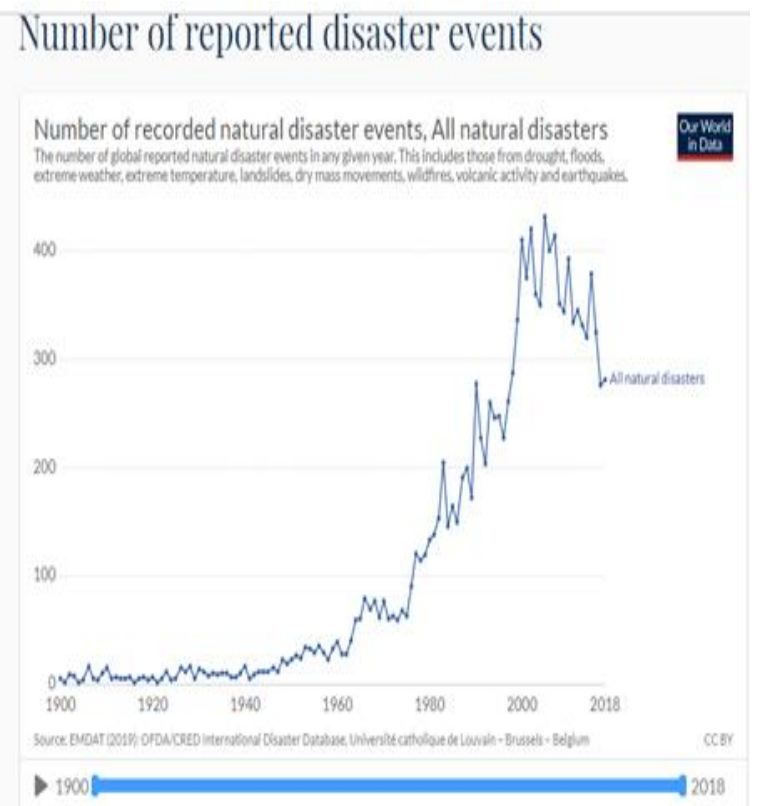

Fig 1: 1 Natural disasters recorded 1900-2018

Since there is this outbreak of disasters in global level, the last decades there are several actions in Greece (Organizations and European programs implemented by Public sector) dealing with the management of disaster results, such as "OASP". (Organization for Forecasting and Protection from Earthquake), "Patch" (European Cultural Heritage Protection Program), etc. [6]. Finally, at an international level, UNDRR was established in 1999 as a secretariat dedicated to facilitate the implementation of the ISDR-International Strategy for Disaster Reduction [20].

As "Disaster Recovery Plan" (DRP) is defined the management policy and procedures used to guide an enterprise response to a major loss of enterprise capability or damage to its facilities. The DRP is the second plan needed by the enterprise risk managers and is used when the enterprise must recover (at its original facilities) from a loss of capability over a period of hours or days (NIST [4]).

Because of the importance of preventing registry corruption and ensuring the continuity of operation of an administration unit, the concept of "IT DR" (Information Technology Disaster Recovery) is of special importance for IT professionals and administrators [10]. An "IT DR" project is a plan for recovering the files of an institute, following such events and aims to increase the chances of normal reinstatement of operation and reduce the risk of data loss [11]. "Ready" is a national public service campaign founded in 2003, and designed to educate and empower the American people to be prepared, respond to, and mitigate emergencies, including natural and man-made disasters. The goal of the campaign is to promote preparedness through public involvement [12].

At the core of the most successful public or private sector units is always a high quality, up-to-date, well-maintained information system. As the present paper refers to information systems used in public services, it would be a mistake not to refer to the term e-Government (Electronic Government) [22]. E-Government contributes to the state modernization, providing higher quality and high value services, making more efficient use of available resources and human resources, and reducing costs and time required to complete such requests [23] 


\subsection{Cloud Computing Technology}

Cloud computing is called the on-demand, centralized computing resources (such as network, servers, applications and services) with high flexibility, minimum user effort and high automation (NIST-National Institute of Standards and Technology, 2011) [4] [17]. When using a "cloud", data and software are stored and processed online, via remote computers located in a central database (Datacenter).

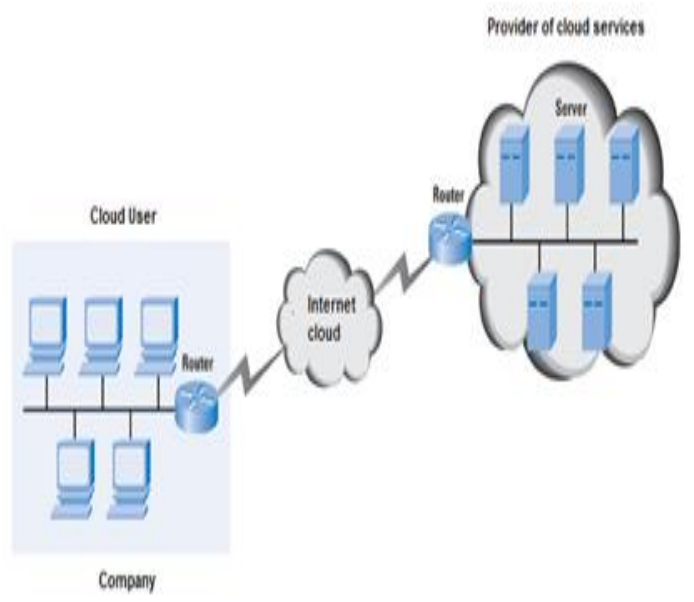

Fig: 2 Computer cloud structure

Cloud Computing refers to applications, which are delivered as services, via the World Wide Web to computer hardware and software located in an information center, which constitute the provider of these services

More analytically, in cloud computing there are the following standards (NIST, 2011):

- Saas (Software as a Service): the applications are provided to the user through the provider's infrastructures.

- Paas (Platform as a Service), the infrastructure provided to users, who themselves develop the software to use.

- IaaS (Infrastructure as a Service): the provider provides computing resources and the network, where the client can develop software and have control over all applications, even the operating system.

Cloud computing services development models are:

1. Public Cloud: the structure of this type makes its resources available to all Internet users.

2. Private Cloud: its structure is "closed" and all its infrastructure, services and files are available to authorized users under an agreement of an organization.

3. Community Cloud: its structure is common to some user groups that belong to a particular community with common interests and needs.

4. Hybrid cloud: The structure of this cloud is the result of a combination of two or more of the above types of clouds (Cloud Security Alliance, 2009).

In the frame of the Greek Public Administration modernization and the implementation of e-Government and administrative reconstruction, actions are constantly being taken to change the structures of public services and reform them in the frame of implementing European and National policies [2]. In the framework of the "82nd International Exhibition of Thessalonica", "Information Society S.A." presented the Government Cloud Computing infrastructure (Government Cloud or G-Cloud), the most up-to-date computing center of Public Administration, based on Cloud Computing \& Virtualization technologies, which is already in full operation since January 2017, and also provides Service Level Agreement (SLA: Service Level Agreement). Electromechanical infrastructure of G-cloud is equipped with dual power and cooling supplies, providing $99.982 \%$ availability $(<1.6$ hours down time), while computing (servers, storage, network, security) has designed with an architecture which allows uninterrupted operation, creating an efficient, easily manageable, stable and secure environment, with its availability reaching $99.75 \%$. All the security standards taken in mind during the implementation of GCloud comply with ISO 27001: 2013 security standard. The aim of G-Cloud is to share computing infrastructures with public administration organizations, thereby reducing their procurement, maintenance and technical support cost. At the same time, the use of the G-Cloud by a Public Agent will offer it flexibility and speed in the management of computing resources (immediate expansion and reduction, depending on the requirements of the organization), guaranteed availability of the provided services G-Cloud thanks to SLA and highlevel security of applications and data.

\section{PROBLEM WORDING}

The administrative unit under consideration in the present paper is a secretariat of a University Department (Secretariat of the Department of Industrial Design and Production Engineering, School of Engineering, University of West Attica) and its operation is based on a combination of files in electronic and printed form. The whole registry is made up of many entities in printed and electronic form, but during the stage of recording the operation of the administration unit, it was necessary to simplify the characterization of the entities, so as to be mapped and coded during the analysis stage, and at a possible future development of the information system. At this point, it should be noted that the main issue is the development of an information system that provides a way to recover after a possible disaster and not the complete mapping of the under study unit.

The following categories are related to the archive of the secretariat:

1. Archive of incoming documents (e.g. administrative documents, student applications, applications from members of the teaching staff, etc.).

2. Archive of outgoing documents, signed and stamped (e.g. administrative documents, certificates, etc.).

3. Archive of grade lists.

4. Individual folders of students and graduates.

5. Registry books of students and graduates.

At a first view, some of the disadvantages /dysfunctions that result from the daily use of documents in printed form:

- Difficulty to quickly locate a document, as space is not sufficient for optimal placement and therefore delay in implementing daily tasks and providing relevant services.

- Risk of loss or damage of the printed material.

- Inadequacy to keep back-up in a form that ensures the recovery after a possible disaster.

- Spatial problem due to the large mass of the file in printed 
form.

The basic applications and types of files used for the everyday operation are:

1. Electronic Application for Management of student issues: It is an application, which is used for the management of databases and following they can be extracted reports and statistics. It is about the most essential tool for the providing of services to students.

2. Electronic Application for Electronic Protocol of Incoming-Outgoing Documents: this application is used for the electronically recording and assigning a Protocol Number to the documents and for their processing.

3. Email: Any user can log in to email, via a web browser or through an installed email management application (e.g. Outlook Express or Microsoft Outlook).

4. Electronic Document File: It is about files in editable and non-editable form (word / excel documents, pdf scanned documents or documents extracted from the electronic protocol).

As it can be seen from the above, for the proper operation of the unit, under the current conditions, the required infrastructure must involve:

- Sufficient space for keeping the printed file (documents, personal folders of students/graduates, etc.).

- Computers and peripherals for access to computer applications.

- Storage devices for file backup.

File backup is still kept with a relatively complex system, as not all files are in a unique storage area. For the documents registered in the electronic protocol, as well as the data registered in the Electronic Application for Student Management, the backup of files is kept on central servers located at another building of the University premises. For any other documents, mainly in editable form are used portable storage devices (optical drives, flash memory sticks, etc.).

More specifically, the methods used for file backup are represented in the following text:

File of incoming documents: These documents are kept as electronic files in the electronic application (electronic protocol). The backup of this application is kept on a central server.

Archive of outgoing documents (signed and stamped): These documents are kept as electronic files in the electronic application (electronic protocol). The backup of this application is kept on a central server. For this type of document, however, keeping a genuine original is important.

Archive of grade lists: Student grades are registered in the Electronic Application for Student Management, but in this case (similarly with the outgoing signed documents), keeping the original in printed form is of utmost importance. The backup of the Electronic Application for Student Management is stored on a central server.

Individual folders of students and graduates: Personal documents and grade lists of students are registered in the relevant computerized application, but in this case, keeping a prototype of the personal documents of students in printed form is important.
Registry books of students and graduates: These documents are kept as electronic files in the electronic application (electronic protocol). The backup of this application is kept on a central server.

Electronic Application for Student Management: The backup of this application is kept on a central server.

Electronic Protocol: The backup of this application is kept on a central server.

E-mail: Copies of incoming and outgoing e-mails are by default stored in the email account, where all the users have access through a browser or email management application (e.g. Outlook Express, Microsoft Outlook, etc.). File backup is kept by users in storage devices, which remain in the unit's premises.

Electronic Document File (in editable form, word, excel, scanned in pdf form, etc.): File backup is kept by users in storage devices, which remain in the unit's premises.

As it arises from the above mentioned ways which are used for file backup, it is obvious that is relatively complex, something which resulting from a combination of the different form of files (printed and digital), and the operation of the unit, which is supported by a combination of different electronic applications.

\section{STUDY ANALYSIS OF THE PROPOSED INFORMATION SYSTEM}

After analysing the technical parameters of the above mentioned system, which is applied for a long period, there were located the points which may be improved and constitute the base for the proposed system. The suggestion of a new system is done only in a theoritical basis, taking into account the improvement of the administration unit's operation by applying the current technological advancies and the management strategies, and there is no proposal for replacing or unifying the existing information systems.

More analytically, after recording the current situation of the administration unit and taking into account the following points:

- the current trends of technology government

- the necessity to implement an information system that will ensure the immediate recovery of the service's operational data in case it arises a disaster,

- the data derived from the bibliographic review, investigating this specific issue

- the provisions of the Public Service Communications Code on document management,

so as to to design the proper solution, which is appropriate for the situation was recorded and in accordance with the strategies which must be followed. According to the Public Service Communications Code [8]: it is well known that the large volume of documents handled by employees of the public services and the total lack of management systems, in combination with the limited and ever-shrinking storage space, make imperative the necessity to take action and proceeding in a new integrated system. The whole study of the system was conducted towards the implementation of an Integrated Electronic Document Management System, which will ensure: 
- Sorting the documents with every field and attribute of the file

- Searching of documents with multiple ways and criteria

- Retrieving documents whose content is relevant to the search expression

- Display document information with flexible sorting criteria (e.g. category, protocol number, date)

- Display of the document in summary or in full view

- Flexible configuration of the user display

- Importing of all documents through scanner, word processors and spreadsheets, so as all the files will be in digital form

- Compression of images for efficient storage management

- Monitoring of the documents' flow

- Support different levels of access per user

- Providing a user friendly graphical interface

- Possibility to send the document to a user or group of users

- Ability to record user activities history

- Ability to select action from a customized list

- Ability to connect to a digital signature subsystem

The solution, which is configured as a result of the bibliographic review, internet research and the study of the administrative unite operation is summarized in the following two steps:

1. Full digitization of all printed material, and more specifically: the documents of student files, all the signed documents, certificates issued by the secretariat, etc., it is obvious that to keep copies of printed material would not be a cost-effective solution and simultaneously wouldn't conform with the ecological spirit that exist in all institutions, public and private, over the last decades.

2. Implementation of an information system based on a cloud computing platform, as the current backup method was imprinted during the recording, it was considered non-effective, therefore it is suggested a standardized way of file backup in a unified space, after the stage of full digitization.

In the frame of the present project it was done a recording of the administration unit's arrangement of registry and the way of its operation, but in case such a system will be developed will be needed an in-depth analysis. It is suggested the investigation of the following points:

1. The full illustration of the existent system's architecture and the technical approach of the transition to the new system, so in case the solution is not the appropriate for the specific infrastructures not to proceed to the next stages. According to the internet research was conducted and the analytical examination of the data collected, the transition to cloud computing technology is permissible.

2. The individual technical features relating to network infrastructures, such as bandwidth (within the cloud, as well as between the cloud and other services). This issue doesn't seem to constitute any problems in the implementation of the new system.
3. Processing capacity of existing resources: possible upgrade need.

4. Checking provider credibility.

5. Checking of Security offered (e.g. multifactorial authentication, encrypted data transfer, etc.).

6. Checking offered data confidentiality and privacy (identifying the ways of providing safeguard).

7. Adaptation of the provider to the regulatory requirements of the University, in accordance with the current legislation and the relevant regulations.

8. Determination of the accessibility of users to the new system. In order to safely operate the new system, it is imperative to implement a user management subsystem. It is a discrete subsystem that addresses the management of access, roles, information available and the functionality that will be available to any user of the system / subsystem. User authentication is configured by the system administrator.

9. Staff training (to the new commands, displays, and the new philosophy of cloud computing).

10. License Cost of Cloud Computing Platform. If the system is outsourced, the biggest expense will be the cost of the agreement with the provider. Typically, service providers have detailed financial models, and it is used to cost depending on the usage of the platform services (Khajeh-Hosseini et al., 2010). There are different costing models available in the market for such services. However, the most common model of pricing is that of Armbrust, which a short-term cost model is. Researchers have analyzed the financial issues raised by Cloud Computing and analyzed them from two perspectives. One for the consumer-user and one for the provider. Both purposes have different price / cost models.

11. Cost of transition from one system to another and maintenance costs.

12. Costs of documents scanning, as it requires numerous working hours.

The cost of the consequences of a natural disaster is inestimable, so a solution which is so intact, apart from the implementation of prevention strategic and full automation will certainly yield a profit. The initial purpose of this paper was to identify alternatives, so that the most cost-effective option to be selected based on the needs of the administration unit. However, by analyzing the resources provided and the current technology trends, the alternatives were rejected, as they were not as cost-effective as the proposed one. The proposed solution was evaluated on several criteria, such as:

- The required time for the implementation (hardware digitization and cloud computing platform)

- The convenience of staff training: according to relevant research, staff training will not be time consuming, as they are already familiar with electronic applications

- Implementation costs

- $\quad$ Saving resources over the current system

- Providing security to users (from viruses and malware) at a relatively low cost.

A key role has been played by the fact that, as mentioned in the previous chapter, information systems of this type are 
already being implemented in the wider public sector (Gcloud example). In addition, Public Administration in Greece is moving towards full automation of services and full registration of data on the internet. The objectives identified on the basis of the results of the implementation study are as follows:

- More efficient module operation - easier access to files, as they will all be available digitally in the cloud area (now the employee will be able to search the scanned file rather than the physical folder).

- Recovery of electronic files in case of natural or manmade disaster.

- Greater efficiency in shared resources.

- Low system maintenance requirements.

- Towards the achieving of the aforementioned objectives, it's impossible not to keep in mind that the main problems that e-government faces in Greece are the following [1]:

- Difficulties in adopting Organizational Information Models

- Fragmented standardization efforts

- Lack of common standards and interoperability of systems

- manning of the public services in not adequate

- Low efficiency of information systems due to the aforementioned reasons.

\subsection{Implications and risks of integrated the proposed system}

Apart from the positive effects of implementing the proposed system, the main risk is the potential infringement of access and intrusion by malicious users as the system is internet based. However, as this risk is taken into account when designing and developing the system, the danger is minimized through the use of very robust programs that protect data against malicious internet users and any other threatens.

\section{CONCLUSIONS}

It is evident from the description of the administration unit's operation that the existing system is quite 'weak' to confront the consequences of a potential disaster, and in particular if the size of the disaster is extensive, the problem is getting worse, as the central servers as well as the alternative storage devices, are in the same premises. The basic concept of the proposed solution is the supporting of the normal operation of the administration unit after a possible disaster.

As it arises from both the existing system and the corresponding information systems, like the one proposed, have already implemented to institutions/units, it is absolutely determined that full automation and implementation of cloud computing will have many advantages. The most important of them is the contribution to the continuous normal operation of the unit, the increasing of the efficiency of the employees and finally it will be a strong response to the potential danger of natural or man-made disaster.

Even if the unit is damaged, the recovery will be achieved quickly, as long as the files will be derived from the storage area of the cloud. At the same time, digitization enables the archive to be unified and gradually reduces the demands on physical storage, while reduced print requirements are in line with the ecological spirit of the University as a whole.

In addition, it is very important that the proposed solution is in line with European directives on public administration, which promote enhanced e-government, minimize resource expenditure and improve interoperability.

\section{REFERENCES}

[1] Efthimios L. Lekkas, Natural and Technological Disasters, National and Kapodistrian University of Athens, 2000, ISBN: 960-90329-0-7

[2] Savvatou Tsolakidou, Effective Management of Organizational Change, National Center for Public Administration and Local Government, 2015.

[3] Mitilessis Nikos, Total Quality Management in the Public Sector and its Implementation in local Government, Diploma Thesis, University of Peloponnese, 2016.

[4] National Institute of Standards and Technology https://www.nist.gov

[5] Ali Khajeh-Hosseini, Ian Sommerville, Ilango Sriram, Research Challenges for Enterprise Cloud Computing, 1st ACM Symposium on Cloud Computing, SOCC, 2010.

[6] Susan Duhl, Maria Lyratzi, Anna Petropoulou, Disaster Management of Cultural Institutions - Brief Overview in the US, Europe and Greece, (available at the webpage https://www.slideshare.net/disasterplangr/ss-48189480 $(22 / 8 / 19)$

[7] Galazoudi Erasmia, Evolution and trends of bibliography in natural disaster research, National and Kapodistrian University of Athens, 2016.

[8] Public Services Communication Regulation, Ministry of Interior, 2003.

[9] Tielan Zhua, Yongqiang Xieb et al., IT Disaster Tolerance and Application Classification for Data Centers, International Congress of Information and Communication Technology, ICICT, 2017.

[10] Shropshire, J. and C. Kadlec, Developing the IT Disaster Recovery Planning Construct, Journal of Information Technology Management, 2009

[11] Hiatt, C.J., A primer for disaster recovery planning in an IT environment, IGI GLOBAL, 2000

[12] READY Campain https://www.ready.gov/about-us

[13] P. Fitsilis, Modern Business Information Systems, 2015 (available at the webpage https://creativecommons.org/licenses/by-nc-sa/3.0/gr/ )

[14] Shakar Bandi, Mallikarjun Angadi and J. Shivarama, Best Practics in Digitisation: Planning and Workflow processes, International Conference on the theme 'Emerging Technologies and Future of Libraries: Issues and Challenges', Gulbarga University, 2015

[15] Dandoulaki Miranda, Kallikratis - Civil protection and local government, EETAA

[16] Philip B. Crosby, Quality Is Free: The Art of Making Quality Certain: How to Manage Quality, 1979, available at the webpage (https://www.referenceforbusiness.com/management/Pr$\mathrm{Sa} /$ Quality-and-Total-Quality-Management.html ) 
International Journal of Computer Applications (0975 - 8887)

Volume 177 - No. 23, December 2019

[17] National Research and Technology Information System http://www.epset.gr/el/content/ypologistiko-nefos-cloudcomputing

[18] Ralph Stair, George Reynolds, Principles of Information Systems, Course Technology, Cengage Learning, 2010.

[19] Qusay f. Hassan, Demystifying Cloud Computing, 2011, (available at the webpage https://www.researchgate.net/publication/215795101

[20] UNDRR United Nations Disaster Reduction https://www.unisdr.org/who-we-are

[21] Dedes Argyrios, Cloud Computing and Intelligent
Techniques: Overview and Applications, University of Piraeus, 2016

[22] Ministry of Education, Research and Religious Affairs Open Governance

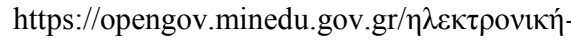

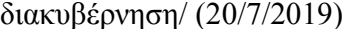

[23] D. Spinellis, N. Vasilakis, et.al, E-Governance in Greece: Successes, Problems, and the Road to Digital Transformation, 2018, "Dia-Neo-sis, Research and Analysis Organization", 2018

[24] Leonidas S. Chitiris, Loukas N. Anninos, Management and Quality of Services, 2015 